\title{
Macroscopic identification of early myocardial infarction by dehydrogenase alterations
}

\author{
REUBEN A. RAMKISSOON \\ From the Department of Pathology, Memorial Hospital of Long Beach, Long Beach, \\ California, U.S.A.
}

SYNOPSIS A method utilizing a general dehydrogenase reaction has been used to demonstrate early gross myocardial infarctions. The procedure takes advantage of substrate and enzyme loss from the damaged myocardium. In the viable muscle, where endogenous substrates, coenzymes, and dehydrogenases are present, reduction of Nitro-BT yields a dark blue formazan. Necrotic muscle fibres remain unstained by this technique.

A survey of 31 human hearts obtained at necropsy disclosed that there is no alteration in the Nitro-BT reaction following acute coronary insufficiency with sudden death or severe congestive heart failure. The earliest myocardial infarct to show loss of dehydrogenase activity was of eight hours' duration. Post-infarction scars and patchy interstitial fibrosis provided very precise information concerning topographic relationships when this method was applied to heart slices.

It is frequently difficult to identify early myocardial infarction at necropsy. Unequivocal gross anatomical changes are not evident for $\mathbf{2 4}$ to $\mathbf{4 8}$ hours following the occlusion of a major coronary artery in man (Gould, 1960; Mallory, White, Salcedo-Salgar, 1939). Furthermore, even though minimal histopathological evidence can be seen as early as six hours, the diagnosis may be missed in the absence of gross anatomical changes because the random areas examined histologically may not show the infarct.

In the recent literature, both experimental and clinical reports indicate that several enzymes appear in the blood soon after myocardial infarction, and that concurrently there is a decrease of these enzymes in the infarcted myocardium (Nydick, Wróblewski, and LaDue, 1955; Graves, Koepke, LaFond, and Ross, 1962). Wachstein and Meisel (1955) found a drop in succinic dehydrogenase activity in ischaemic myocardium in six to eight hours. Neoral and associates (Neoral, Kolin, Kodousek, and Kvasnicka, 1956; Kolin and Neoral, 1960) used sodium tellurite as an oxidation-reduction indicator for the macroscopic identification of early myocardial infarction. In 1963, Shnitka and Nachlas reported an early loss of labile substances from ischaemic heart muscle responsible for differential staining with a dehydrogenase reaction.

With the foregoing information available, it Received for publication 15 February 1966. seemed worthwhile further to investigate the possibility of using an enzyme reaction for the gross identification of early myocardial infarction. Accordingly, a histochemical method was applied to large transverse slices of human heart muscle in attempts to visualize the sites of activity of several endogenous substrates, coenzymes, and dehydrogenases in normal and ischaemic myocardium.

\section{MATERIAL AND METHODS}

Transverse slices of human hearts were obtained at necropsy from 31 patients with arteriosclerotic cardiovascular disease. These were obtained within two to 48 hours after death.

Prior experience by others (Neoral, et al. 1956; Shnitka and Nachlas, 1963) showed that of the dehydrogenase reactions, no one method in particular was strikingly superior to the other for the gross demonstration of early myocardial infarctions. The incubation solution utilized in this study for the macroscopic identification of early myocardial infarction consisted of Sorensen's phosphate buffer $(0.1 \mathrm{M}), p \mathrm{H} 7.4$, and $0.5 \mathrm{mg}$. $/ \mathrm{ml}$. of Nitro-BT (2,2'-di-p-nitro-phenyl-5,5'-diphenyl-3,3'-(3,3'-dimethoxy $-4,4^{\prime}$-biphenylene) ditetrazolium chloride). For convenience, stock solutions were kept consisting of phosphate buffer at $1 \mathrm{M}$ concentration and Nitro-BT at $5 \mathrm{mg}$. $/ \mathrm{ml}$. When ready for use, the incubation solution was prepared by mixing 1 part buffer, 1 part Nitro-BT, and 8 parts water.

The slices of hearts were rinsed in running cold water to remove blood and tissue juices from the cut surfaces. 
These were then placed in the buffered tetrazolium solution for a 30 -minute incubation in a $37^{\circ} \mathrm{C}$. water bath. Normal myocardium stained dark blue within 15 minutes, while infarcted myocardium remained unstained, or faintly stained.

The effect on the differential staining reaction by the loss of endogenous substrates and coenzymes from early infarcts was assessed by adding succinate $(0 \cdot 1 \mathrm{M})$ to the standard buffered Nitro-BT solution. This was useful for hearts that were removed from embalmed cadavers after a post-mortem interval of more than six hours. The succinate was added to compensate for loss of endogenous substrate from normal heart muscle. Hearts obtained from cadavers refrigerated at $6^{\circ} \mathrm{C}$. for as long as 48 hours gave satisfactory results. Embalming procedures precluded valid differential staining reactions although useful topographic information was obtained if the heart was examined within two hours after embalming. Trochar embalmed hearts were unsatisfactory (Shnitka, 1963).

After the histochemical reaction employing Nitro-BT had differentiated normal from necrotic myocardium, it was possible to correlate the gross findings with clinical and electrocardiographic evidence available on each patient. Histological correlation will be a subject of a subsequent report.

The necropsy cases were divided into groups on the basis of the clinical histories, as follows:

GROUP I Group I included patients whose clinical histories were suggestive of recent myocardial infarction. There was electrocardiographic, laboratory and histopathological evidence of myocardial infarction.

GROUP II Group II comprised patients with known arteriosclerotic cardiovascular disease, but without the usual clinical or histological evidence of myocardial infarction.

GROUP III Group III consisted of patients with other diseases which might be expected to produce secondary myocardial ischaemia during the terminal episode.

\section{RESULTS}

GROUP I Heart slices from 12 patients with proved acute myocardial infarction were studied. Intense staining was noted within 15 to 30 minutes in normal portions of the myocardium. The pigment deposited by the activity of tissue dehydrogenases resulted in the reduction of Nitro-BT to a dark blue formazan, which gave good contrast between active and inactive portions of heart muscle. Infarcted myocardium, on the other hand, showed little or no tissue staining (Fig. 1). The earliest infarct showing loss of tissue dehydrogenase activity was eight hours. Older lesions consisting of 'recent infarction' and patchy interstitial fibrosis were also clearly demonstrated by this staining reaction.

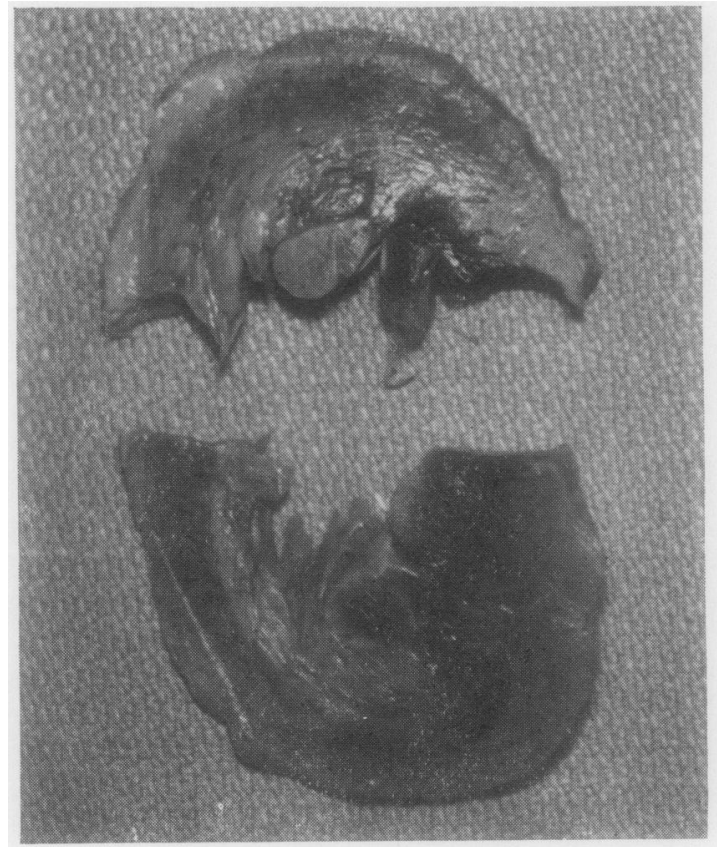

FIG. 1. Acute myocardial infarction that is grossly visible. Note loss of dehydrogenase activity (no colour development) in the infarcted areas.

GROUP II Of the 15 patients with arteriosclerotic cardiovascular disease in this group, there were none who showed the usual clinical symptoms of myocardial infarction. However, in each case there were areas of myocardial ischaemia or necrosis which were delineated by little or no colour development in the areas of infarction (Fig. 2).

GROUP III Heart slices from four patients comprised this group. Correlation of the clinical, gross necropsy, and histochemical reactions revealed no staining or light staining areas of ischaemia. These subsequently disappeared with additional substrates and a 30 - to $45-$ minute incubation at $37^{\circ} \mathrm{C}$.

\section{DISCUSSION}

Because of the minimal histological changes present in ischaemic myocardium for six hours or less, many investigators have been stimulated to search for more easily recognizable histochemical changes (Govier, 1945; Cummings, 1960). It has been shown that, after coronary artery ligation in a dog, assays of myocardium from suspected areas of infarction revealed little or no loss of enzyme activity initially. However, within one to four hours, depletion of enzymes in the ischaemic myocardium begins. The 


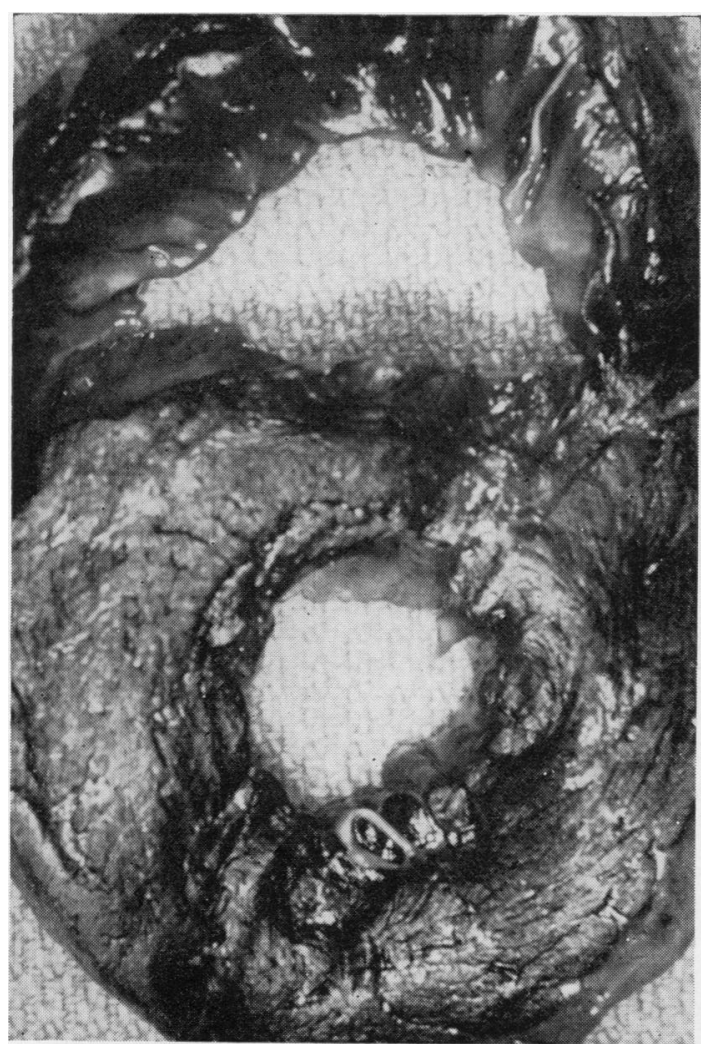

FIG. 2. Early myocardial infarction, anterior wall and septum, with postero-lateral ischaemia approximately of eight hours' duration, not grossly visible without dehydrogenase histochemical staining.

simultaneous rise of these enzymes in the blood is regarded as evidence of irreversible myocardial damage (Hamolsky and Kaplan, 1961; Strandjord, Thomas, and White, 1959; Ruegsegger, Nydick, Freiman, and LaDue, 1959). Gross recognizable anatomical alterations in the myocardium, on the other hand, do not become evident for as late as 12 to 24 hours after coronary artery ligation or occlusion. It is for these reasons that histochemical techniques utilizing enzyme changes for the early identification of gross myocardial infarction are of value.

Neoral and his associates (Neoral et al., 1956; Kolin and Neoral, 1960) applied their tellurite reaction to the hearts of 20 patients with suspected or proved myocardial infarction. The earliest infarct which they recognized was in a patient who died seven hours after the onset of clinical symptoms.
There were neither gross nor microscopic findings. Cain and Assmann (1960) reported 14 human hearts stained at necropsy with neotetrazolium chloride. This histochemical reaction served accurately to outline areas of acute and subacute infarction, interstitial myocardial fibrosis, and post-infarction scars. More recently, Shnitka and Nachlas (1963) surveyed 23 human hearts treated with Nitro-BT solution. The earliest myocardial infarction to show loss of dehydrogenase activity was of eight hours' duration.

In this study of 31 human hearts, reduction of buffered Nitro-BT yielded a dark blue formazan in normal myocardium. Necrotic muscle fibres remained unstained due to the loss of endogenous substrates, coenzymes and dehydrogenases in the infarcted tissue. Ischaemic myocardium was faintly stained but with the addition of substrates and a longer incubation period, differential staining with formazan disappeared. This was interpreted as substrate deficiency in ischaemic myocardium and true enzyme loss in the necrotic muscle. This method gives precise information concerning the gross topographic anatomy of myocardial infarction and its arterial blood supply, electrocardiographic changes, and biochemical characteristics (Jennings and Wartman, 1957a and b; Abildskov, Wilkinson, Vincent, and Cohen, 1961). This technique is more sensitive and convenient than the tellurite method because results can be obtained in 30 minutes in contrast to three to eight hours.

\section{REFERENCES}

Abildskov, J. A., Wilkinson, R. S. Jr., Vincent, W. A., and Cohen, W. (1961). Amer. J. Cardiol., 8, 485.

Cain, H., and Assmann, W. (1960), Klin. Wschr, 38, 433.

Cummings, J. R. (1960). Circulat. Res., 8, 865.

Gould, S. E. (Ed.) (1960). Pathology of the Heart, 2nd ed., pp. 599-606 Thomas, Springfield, Illinois.

Govier, W. M. (1945). Amer. Heart J., 29, 384.

Graves, M. D., Koepke, J. A., LaFond, D. J., and Ross, A. (1952). Amer. J. clin. Path., 37, 282.

Hamolsky, M. W., and Kaplan, N. O. (1961). Circulation, 23, 102. Jennings, and Wartman, W. B. (1957a). Med. Clin. N. Amer., 41, 3. $\longrightarrow$, (1957b). Arch. Path., 63, 580.

Kolin, A., and Neoral, L. (1960). Cas. Lék. Čes., 99, 363.

Mallory, G. K., White, P. D., and Salcedo-Salgar, J. (1939). Amer. Heari J., 18, 647.

Neoral, L., Kolin, A., Kodousek, R., and Kvasnicka, J. (1956). Acta Univ. palack. olomuc., 11, 127.

Nydick, I., Wróblewski, R., and LaDue, J. S. (1955). Circulation, 12, 161 .

Ruegsegger, P., Nydick, I., Freiman, A., and LaDue, J. S. (1959). Circulat. Res., 7, 4.

Shnitka, T. K. (1963). Personal correspondence.

- and Nachlas, M. M. (1963). Amer. J. Path., 42, 507.

Strandjord, P. E., Thomas, K. E., and White, L. P. (1959). J. clin. Invest., 38, 2111.

Wachstein, M., and Meisel, E. (1955). Amer. J. Path., 31, 353. 